\title{
Clinical Versus Public Health Perceptions of Notifiable Disease Reporting Burden
}

\author{
Debra Revere ${ }^{\star 1}$, Rebecca Hills ${ }^{1}$, Shaun J. Grannis ${ }^{2,3}$ and Brian E. Dixon ${ }^{2,3}$ \\ ${ }^{1}$ University of Washington, Seattle, WA, USA; ${ }^{2}$ Indiana University, Indianapolis, IN, USA; ${ }^{3}$ Regenstrief Institute, Indianapolis, IN, USA
}

\section{Objective}

Introduction of new health information technologies can produce unanticipated consequences on existing user behaviors, workflow, etc. Prior to implementing a public health reporting intervention, we conducted a series of interviews regarding workflow and perceptions of task burden with respect to notifiable condition reporting.

\section{Introduction}

The importance transmitting clinical information to public health for disease surveillance is well-documented [1]. Conventional reporting processes require health care providers to complete paper-based notifiable condition reports which are transmitted by fax and mail to public health agencies. These processes result in incomplete reports, inconsistencies in reporting frequencies among different diseases and reporting delays [2] as well as time-consuming follow-up by public health to get needed information [3]. One strategy to address these issues is to electronically pre-populate report forms with available clinical, lab and patient data to streamline reporting workflows, increase data completeness and, ultimately, provide access to more timely and accurate surveillance data for public health organizations.

Prior to implementing an intervention that includes using pre-populated forms, we conducted interviews in clinical and public health settings to identify the barriers and facilitators to adopting and utilizing the forms and their potential impact on workflow and perceived burden. These interviews are a component of a larger mixed methods evaluation that will triangulate pre- and post-intervention quantitative data quality measures with qualitative results.

\section{Methods}

Semi-structured interviews were conducted between 09/2012 and 07/2013 with individuals $(\mathrm{n}=11$ ) responsible for submitting notifiable condition reports in 7 outpatient clinics. Semi-structured interviews with public health workers $(n=8)$ in 2 public health agencies were conducted in 09/2013. Interviews focused on facilitators and barriers to the intervention and included questions regarding current reporting practices; perceptions of report completeness, accuracy and timeliness; reporting burden; and concerns and perceived benefits of the pre-populated form intervention.

Interviews were recorded, transcribed and imported into qualitative data analysis software. Two coders thematically coded the transcripts and notes taken during the interviews. The study received joint UW/ IU Institutional Review Board approval.

\section{Results}

Perceptions of burden differ between clinical and public health settings. In the clinical setting, reporting processes are perceived as minimally disruptive to routine workflow and the need to provide supplemental, missing or corrected data is not perceived as occurring frequently enough to be a burden. These results greatly contrast with public health perceptions of burden. Incomplete reports, inaccuracies and reporting delays are seen as severely impacting task burden and public health workers' ability to interpret disease incidence and make comparisons among public health jurisdictions. While it was anticipated that the intervention could potentially reduce processing burden and provide higher quality surveillance data, some public health respondents expressed concerns regarding capacity for processing a possible increase in number of reports post-intervention.

\section{Conclusions}

It is important to identify and address anticipated and unanticipated consequences and their impact on workflow and task burden to ensure health information technology interventions are adopted and utilized in ways that improve individual and population health outcomes.

\section{Keywords}

Public health surveillance; Notifiable condition reporting; Qualitative methods; Public health informatics

\section{Acknowledgments}

This project was supported by Agency for Healthcare Research and Quality (AHRQ) grant \#R01HS020909 (PI: B Dixon). The content is solely the responsibility of the authors and does not necessarily represent the official views of AHRQ.

\section{References}

1. Platt R, Bocchino C, Caldwell B, et al. Syndromic surveillance using minimum transfer of identifiable data. J Urban Health 2003;80:i25-31.

2. Jajosky RA, Groseclose SL. Evaluation of reporting timeliness of public health surveillance systems for infectious diseases. BMC Public Health 2004;4:29.

3. Sickbert-Bennett EE, Weber DJ, Poole C, MacDonald PD, Maillard JM. Completeness of communicable disease reporting. Emerg Infect Dis 2011;17:23-9.

\footnotetext{
*Debra Revere

E-mail: drevere@uw.edu
} 